OPEN

SUBJECT AREAS:

NANOWIRES

SOLAR ENERGY AND PHOTOVOLTAIC TECHNOLOGY

Received

7 October 2014

Accepted

28 November 2014

Published

16 December 2014

Correspondence and requests for materials should be addressed to M.B. (bayindir@nano.

org.tr)

\section{Superenhancers: Novel opportunities for nanowire optoelectronics}

\author{
Tural Khudiyev \& Mehmet Bayindir ${ }^{1,2,3}$
}

'UNAM-National Nanotechnology Research Center, Bilkent University, 06800 Ankara, Turkey, ${ }^{2}$ Institute of Materials Science and Nanotechnology, Bilkent University, 06800 Ankara, Turkey, ${ }^{3}$ Department of Physics, Bilkent University, 06800 Ankara, Turkey.

Nanowires play a crucial role in the development of new generation optoelectronic devices ranging from photovoltaics to photodetectors, as these designs capitalize on the low material usage, utilize leaky-mode optical resonances and possess high conversion efficiencies associated with nanowire geometry. However, their current schemes lack sufficient absorption capacity demanded for their practical applicability, and more efficient materials cannot find widespread usage in these designs due to their rarity and cost. Here we suggest a novel and versatile nanoconcentrator scheme utilizing unique optical features of non-resonant Mie (NRM) scattering regime associated with low-index structures. The scattering regime is highly compatible with resonant Mie absorption effect taking place in nanowire absorbers. This technique in its optimized forms can provide up to $1500 \%$ total absorption enhancement, 400 -fold material save and is suitable for large-area applications with significant area preservation compared to thin-film of same materials. Proposed superenhancer concept with its exceptional features such as broadband absorption enhancement, polarization immunity and material-independent manner paves the way for development of efficient nanowire photosensors or solar thermophotovoltaic devices and presents novel design opportunities for self-powered nanosystems.

$\mathrm{N}$ anotechnology has the potential to fulfill requirements associated with construction of efficient optoelectronic devices through novel material features (i.e. optical and electronic) appearing at the nanoscale. Especially motivation of light-matter interactions in nanowire geometry that can yield absorption comparable with conventional semiconductor films of much greater volume facilitates their in-depth investigation. Particularly, nanowire photovoltaics ${ }^{1-6}$ has been demonstrated to have unique and advantageous features in comparison to its planar counterpart ${ }^{7,8}$ (e.g. using less material, taking benefit of optical resonances and better charge collections). However, single nanowire photovoltaics systems, which have been shown to be useful to supply power for proof-of-concept nanodevices, ${ }^{9,10}$, are incapable to serve as high-power source for more advanced nanosystems. This also limits potential utilization of many nanowire-based photodevices ${ }^{11-16}$, especially those which operate in less dominant (i.e. suffer from low intensity level) radiation zone of solar spectrum (i.e. UV and IR). On the other hand, materials that exhibit better electronic and optical performance than abundant silicon material cannot effectively take place in these nanowire-based designs due to their rarity and cost. Therefore, emerging functionalities and broad application areas (e.g. energy, safety, health) of these photosensors compel creation of these designs with their optimal performances (i.e. regarding to their efficiency, power level, and cost). Especially in nanowire photovoltaics, one of the current focuses is tailoring the light absorption within the nanowire geometry by harnessing distinct engineering strategies. There are significant efforts with satisfied growth towards the maximization of resonant Mie absorption effect by taking the nanowire size and the morphology into account ${ }^{17-20}$. It is also possible to design efficient nanowire solar cells using properly patterned multi-material absorbers ${ }^{21-23}$. Above all, a more prominent way to enhance light absorption within the nanowire scheme relies on utilization of core-shell geometry where both absorber core and transparent shell region induces enhancements via their unique antireflection features ${ }^{24-26}$. Alternatively, plasmonics nanostructures are also tested in the design of efficient nanowire absorbers using their unique light accumulating performance ${ }^{27}$. However, utilization of these engineering techniques still could not provide sufficiently high absorption, power efficiency and large-area applicability that are demanded to be parallel with practicality and cost of nanowire solar cells, hence require further exploration and radical strategies for the handling of these challenging issues.

In this study, we propose a highly-effective and straightforward nanoconcentrator scheme that provide useful solutions regarding the aforementioned issues, such that, in optimized forms of developed technique, it is possible to observe $1500 \%$ overall absorption enhancement, 400 -fold material conservation and over 1.5 -fold area saving in comparison to thin films. Present method utilize the recently described non-resonant Mie (NRM) scattering 

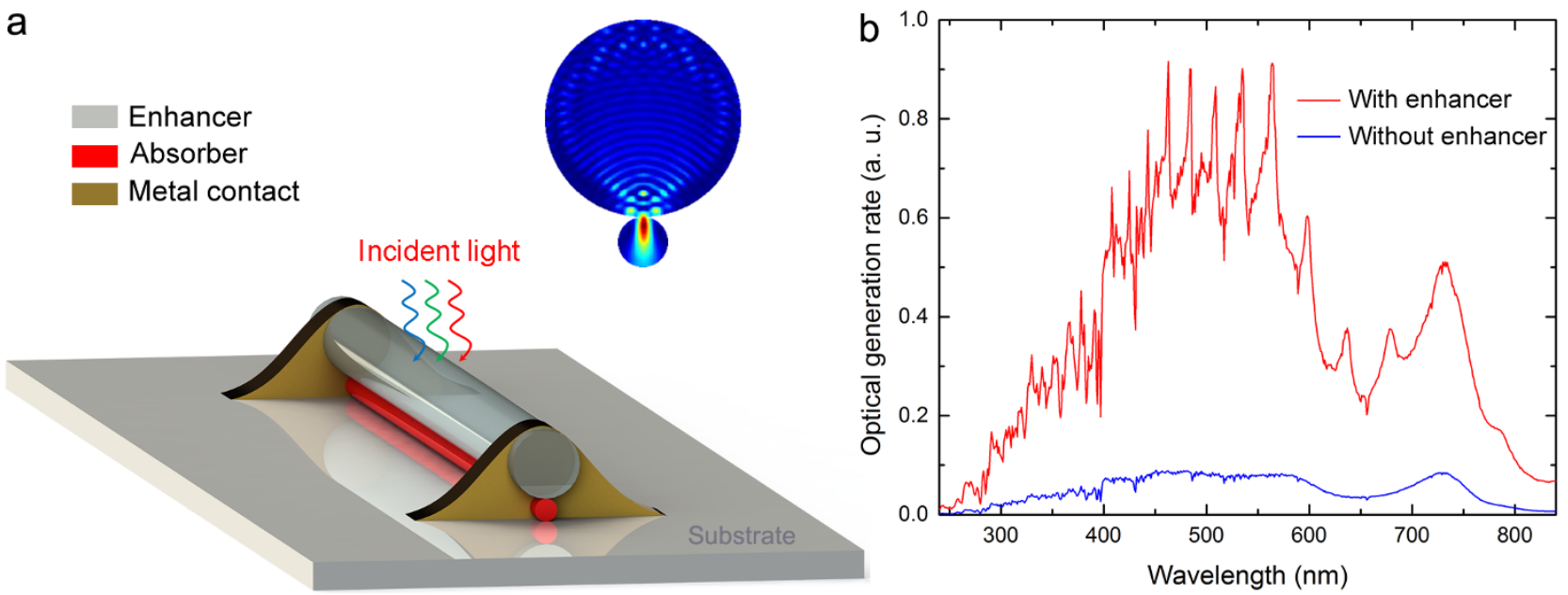

Figure 1 Superenhancer scheme. (a) Proof-of-concept device consist of a-Si absorber nanowire with gold metal contacts on two edges and low-index enhancer wire on it. Inset shows scattered field profile from enhancer that accumulates light inside of resonant absorber nanowire. (b) Absorption capacity of single nanowire is extremely boosted via utilization of our superenhancer wire $(r=1200 \mathrm{~nm})$. Enhancement is effective along longitudinal nanowire axis and at each spectral point of absorption spectrum with comparable enhancement rates. Two broad absorption peaks are induced by resonant Mie absorption taking place in underlying nanowire $(r=100 \mathrm{~nm})$. NRM scattering-based trapping results with conformal, broad and enormous enhancement over bare nanowire absorption profile.

effect $^{28}$ or in its other name anomalous diffraction $z o n e^{29}$, which was found to exhibit superior features that are required by ideal light trapping apparatus (i.e. broadband and polarization independent enhancement, unique field localization and coupling characteristics) and is highly compatible with resonant Mie absorption effect that take place in nanowire absorbers. We call this method as superenhancers regarding these exclusive features and demonstrate its versatile applicability and potential for different design circumstances.

Emerging light trapping scheme: "Superenhancers". Optical properties (i.e. scattering and absorption) observed as a result of lightmatter interactions at nanoscale can be largely explained by the Mie theory ${ }^{30,31}$. In nanowire solar cells, resonant Mie absorption effect is responsible for enhanced absorption and can be engineered for maximizing it taking advantage of leaky mode optical resonances. These resonant points are determined by size of absorber material and strictly depend on incident polarization especially at smaller sizes (Figure S1). Absorption efficiency $\left(Q_{a b s}\right)$ of infinitely long cylinder ${ }^{31}$ can be derived analytically for TE, TM polarizations and unpolarized light (their average) using Mie theory which give solutions as following;

$$
\begin{gathered}
Q_{a b s}^{T M}(x)=\frac{2}{x}\left\{\operatorname{Re}\left(\sum_{m=-\infty}^{m=\infty} b_{m}\right)-\sum_{m=-\infty}^{m=\infty}\left|b_{m}\right|^{2}\right\} \\
Q_{a b s}^{T M}(x)=\frac{2}{x}\left\{\operatorname{Re}\left(\sum_{m=-\infty}^{m=\infty} a_{m}\right)-\sum_{m=-\infty}^{m=\infty}\left|a_{m}\right|^{2}\right\} \\
Q_{a b s}^{u n p}(x)=\frac{Q_{a b s}^{T M}(x)+Q_{a b s}^{T E}(x)}{2}
\end{gathered}
$$

where $\left(x=\frac{2 \pi r}{\lambda}\right)$ is size parameter and $r$ is radius of the core, and coefficients $a_{m}, b_{m}$ appearing in the equations are;

$$
\begin{aligned}
& b_{m}=\frac{J_{m}(n x) J_{m}^{\prime}(x)-n J_{m}{ }_{m}(n x) J_{m}(x)}{J_{m}(n x) H_{m}^{(1)^{\prime}}(x)-n J_{m}^{\prime}(n x) H_{m}^{(1)}(x)}, \\
& a_{m}=\frac{n J_{m}(n x) J_{m}^{\prime}(x)-J_{m}(x) J_{m}^{\prime}(n x)}{n J_{m}(n x) H_{m}^{(1)^{\prime}}(x)-J_{m}^{\prime}(n x) H_{m}^{(1)}(x)}
\end{aligned}
$$

Here, $J_{m}$ represents the $m^{\text {th }}$-order Bessel function of the first kind, $H_{m}$ shows the $\mathrm{m}^{\text {th }}$-order Hankel function of the first kind and the prime indicates differentiation with respect to related functions. These solutions can facilitate understanding of absorption processes within the nanowire geometry ${ }^{19}$ and precisely match with results obtained via finite difference time domain (FDTD) method (Figure S1). On the other hand, scattering of light is useful when it is controlled at a smaller size scale (comparable with incident light wavelength) and can provide a distinct set of useful properties ${ }^{32}$. Scattering efficiency $\left(Q_{s c a}\right)$ of cylinder geometry also can be obtained by the same Mie formalism. Results for TE, TM and unpolarized light cases can be expressed as following;

$$
\begin{aligned}
& Q_{s c a}^{T M}(x)=\frac{2}{x}\left\{\sum_{m=-\infty}^{m=\infty}\left|b_{m}\right|^{2}\right\}, Q_{s c a}^{T E}(x)=\frac{2}{x}\left\{\sum_{m=-\infty}^{m=\infty}\left|a_{m}\right|^{2}\right\}, \\
& Q_{s c a}^{u n p}(x)=\frac{Q_{s c a}^{T M}(x)+Q_{s c a}^{T E}(x)}{2}
\end{aligned}
$$

These two distinct phenomena can be complementary at the sizes where Mie effects are still dominated, such that scattering characteristics can provide desirable light trapping features for the purpose of enhancing resonant absorption capacity of single nanowire ${ }^{33}$.

Intuitively, this scattering regime should be associated with nonabsorptive and low-refractive index nanoparticles. Recently, we investigated scattering properties of low-index nanostructures (i.e. polymer nanowires) in the context of NRM scattering and suggested that this scattering regime has a high potential for various nanophotonics applications. In this interaction domain, light scatters strictly on forward direction and can exhibit focusing feature with $r \gg w$, where $r$ is radius of enhancer and $w$ corresponds to width of scattered light. When these characteristic features (i.e. focusing, unique coupling behaviour and etc.) tailored for optimal resonant Mie absorption conditions (i.e. size parameters, morphology and etc.) a novel light trapping scheme the "superenhancer" effect emerges. During optimization calculations we take absorption enhancement $(A E)$ as a figure of merit and maximize it throughout these processes. $A E$ can be defined as;

$$
A E=\int_{\lambda_{1}}^{\lambda_{2}} A_{e n h}(\lambda) \times d \lambda / \int_{\lambda_{1}}^{\lambda_{2}} A_{\text {bare }}(\lambda) \times d \lambda
$$

where $A(\lambda)$ is absorption spectrum, $\lambda_{1}=240 \mathrm{~nm}$ and $\lambda_{2}=840 \mathrm{~nm}$. Particularly in Figure $1 \mathrm{~b}$ we take solar radiation spectrum, $S(\lambda)$ into account and define optical generation rate enhancement as; 

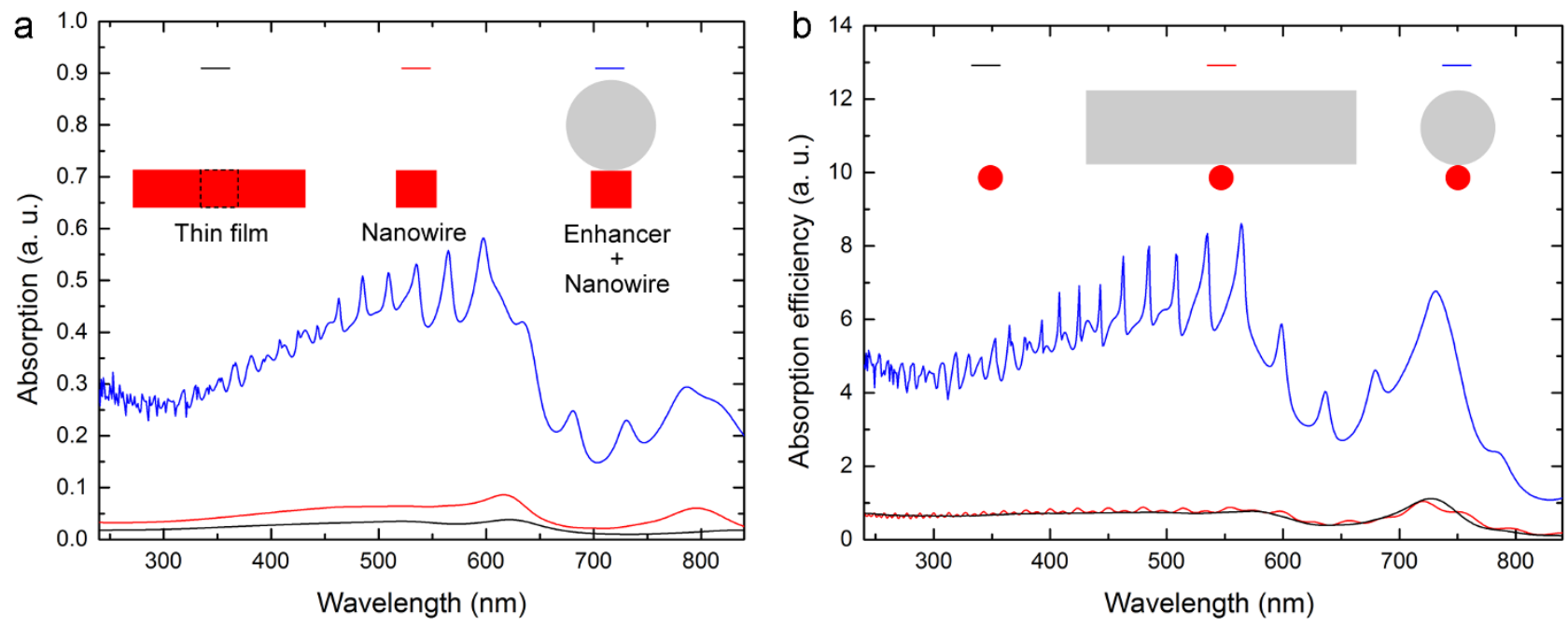

Figure 2 Comparison with thin film effects. (a) New generation solar cells rely on thin film absorber designs. Theoretical calculations are performed to investigate volumetric absorption performance of thin film and nanowire geometry where the latter is observed to exhibit over 2.1-fold better absorption performance. By adding one of the optimum enhancer size $(\mathrm{r}=1200 \mathrm{~nm})$ on it we can get up to 16-fold enhancement in resulting volumetric absorption. This supplies enormous power amplification for same amount of absorber material. For comparison of $1 \mathrm{D}$ nanowire with thin-film scheme, square nanowire is utilized. (b) When thin film effects take place in enhancer region, it is found that enhancement profile will consist of well-known multiinterference peaks on bare absorption profile. We note that similar interference peaks but shifted in their peak positions (due to cylindrical geometry of enhancer) also appear in enhanced absorption spectrum for the presence case of our superenhancer which provide lateral enhancement contribution. This shows that NRM scattering-based effect $(A E \%=700 \%)$ relies on different fundamentals in comparison to well-known interference effect $(A E \%=4 \%)$. During these calculations, materials of nanowire and thin film are selected identically and thicknesses of thin film are defined as magnitude of diameter in nanowire case.

$$
\eta_{O G R}=\int_{\lambda_{1}}^{\lambda_{2}} A_{e n h}(\lambda) \times S(\lambda) \times d \lambda / \int_{\lambda_{1}}^{\lambda_{2}} A_{\text {bare }}(\lambda) \times S(\lambda) \times d \lambda
$$

Both absorption efficiency $\left(Q_{a b s}\right)$ and absorption $(A)$ parameters which are correlated with each other, are used throughout the investigation. Especially, former is useful for demonstration of effectiveness of absorption in given dimensions. During simulations (if not specified apparently) absorber nanowire is selected as amorphous silicon (a-Si) material with $r=100 \mathrm{~nm}$ and enhancer component is defined as transparent material with constant refractive index of $n=1.5$ and size of $r=300 \mathrm{~nm}$. These parameters are chosen for proof-of-concept demonstration which can also be applied to any other cases as long as they remain in size limit and material context of related Mie scattering and absorption phenomena.

Figure 1a represents outline of proposed light trapping scheme. It simply utilizes semiconductor nanowire absorber and low-index nanowire enhancer on it. In resonant effects (such as plasmonics or metallic nanoantenna based trapping techniques) amplified fields are mainly concentrated on edge of metallic structures ${ }^{34}$, therefore resulting enhancement is observed to be highly localized, strictly dependent on type of incident polarization and more importantly resonantly enhance light absorption in specific and narrow wavelength points which have limited overall contribution. However, in NRM scattering-based approach, enhancement conformably add on bare absorption capacity of single nanowire as a result of field focusing feature of scattering regime effective along longitudinal nanowire axis. The broadband enhancement profile observed in Figure $1 \mathrm{~b}$ is associated with utilized scattering regime which is effective within the significant part of solar radiation spectrum. It is worthy to note that, NRM scattering regime is in full accordance with resonant Mie absorption effect, such that coupling of light from enhancer nanowire to absorber does not distort resonant absorption characteristics of bare nanowire therefore it can be easily controlled. During deriving results given in Figure 1b, we select one of the optimal values of enhancer $(\mathrm{r}=1200 \mathrm{~nm})$ for the comparison with absorption spec- trum of bare nanowire that is obtained by considering aforementioned size and material parameters of absorber.

Mie resonances appearing on high-index nanowires can be considered as highly miniaturized analogues of microresonators that is able to trap light resonantly by multiple total internal reflections from the periphery. However due to their small dimensions, nanoscale counterparts are gaining novel functionalities and features (e.g. leaky-mode behaviour, antenna function and etc.). In similar behaviour, our enhancer nanowires can also be viewed as ultimately scaled-down versions of microball ${ }^{35}$ lenses (more precisely cylinder) which is frequently used for the purpose of efficient light coupling in fiber applications. Owing to their small sizes, interaction of the light with our nanowires falls within the domain of wave optics that can be explained utilizing Lorentz-Mie theory, and it gains novel features such as efficient light scattering, focusing of light on contiguous points in contrary to their sub-millimeter scale analogues which dictates focal point arrangements and etc.

In order to gain further insight into effectiveness of proposed technique, we compare both 1D absorber and enhancer with their planar counterparts (i.e. thin film effects) (Figure 2). Light absorption within the conventional thin film solar cell is compared with nanowire geometry of same material $(\mathrm{a}-\mathrm{Si})$ and corresponding radius $(r=100 \mathrm{~nm})$. Absorption efficiencies are found to be superior to their thin film counterparts both for TE and TM polarizations (Figure 2a, Figure S3a). Namely, even though in TE polarization $\left(Q^{T E}=1.5\right)$ nanowire displays lower absorption efficiency in compare to TM $\left(Q^{T M}=3.5\right)$, in both polarization cases nanowire geometry still can provide higher absorption amount $(A E>2)$ than same material and volume of thin film. Therefore, utilizing bare nanowire solar cells together with present technique can result with total $A E$ as high as 16-fold (i.e. proposed technique supplies $A E$ of 8-fold in comparison to bare nanowire absorber where nanowire alone has $>2.1$-fold better absorption performance than thin film). This corresponds to more than one order of magnitude increment in resulting power for same amount of utilized absorber material. It should be noted that, using antireflection coating in thin-film case can boost light 


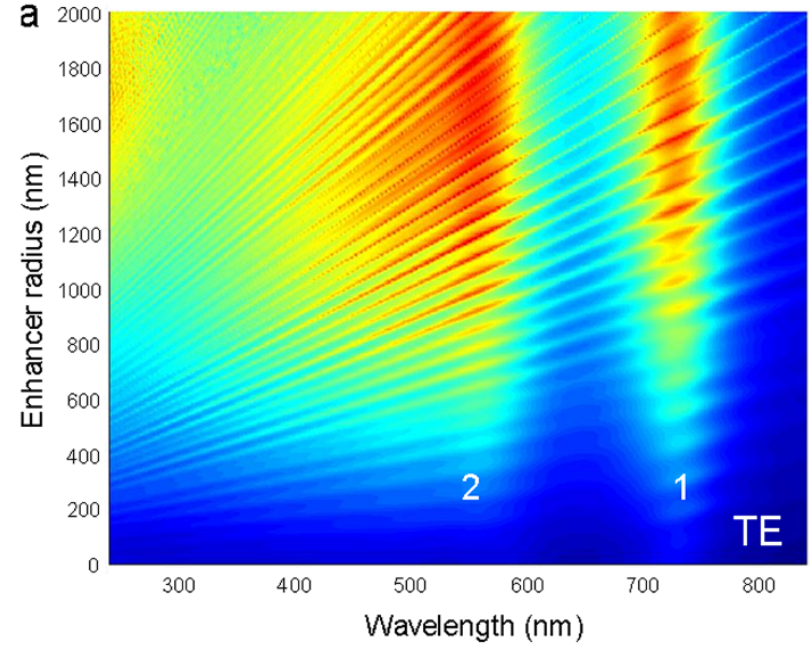

C

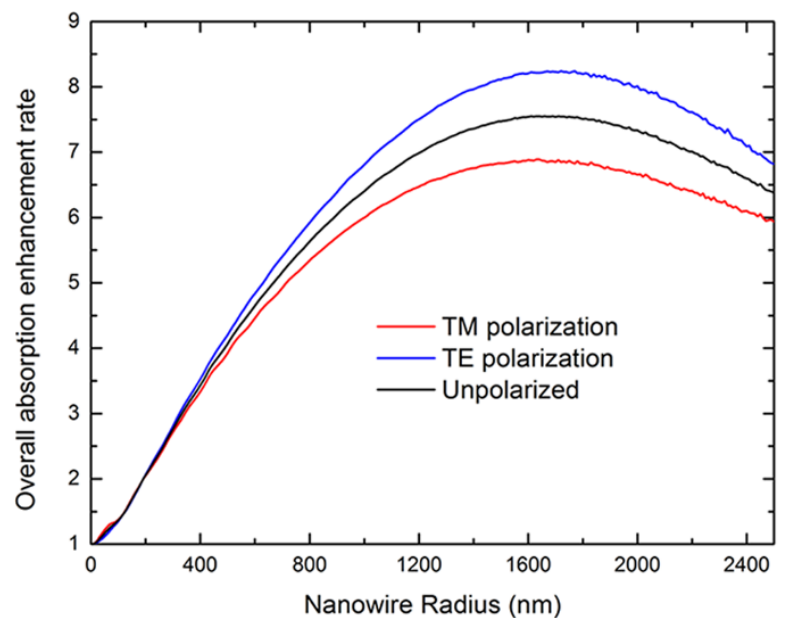

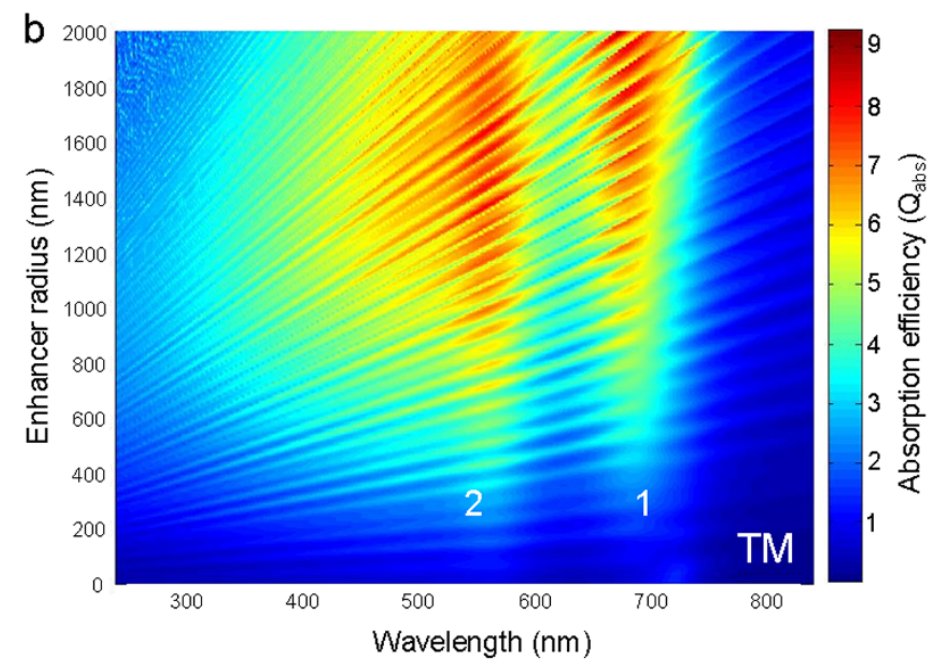

d
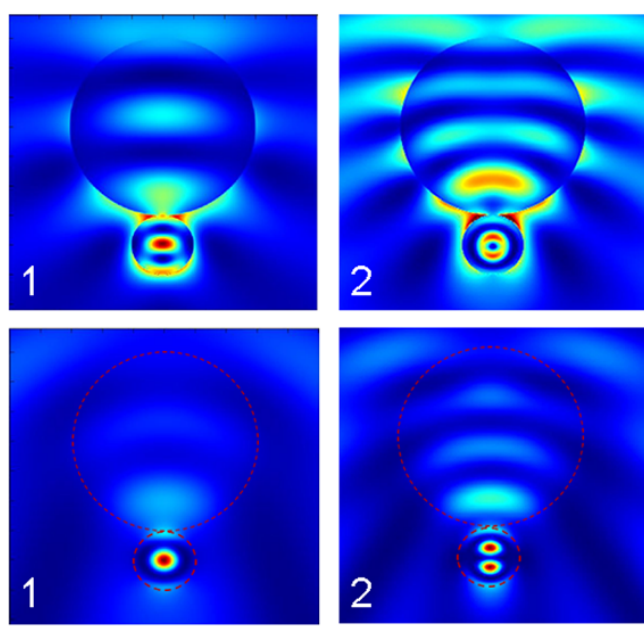

Figure $3 \mid$ Coupling of NRM scattering to resonant Mie absorption. (a,b) Light scattering from low-index enhancer boosts light absorption with sizedependent manner. NRM scattering spectrum encompasses a broad wavelength range matching with absorption spectrum of nanowire absorber. As a unique feature of NRM scattering, absorption enhancement is polarization independent; therefore, it significantly contributes especially to low absorption efficiency of the nanowire in TE polarization case. (c) Optimum radial size for maximum AE is found to be around $1500 \mathrm{~nm}$. However different size regimes can be applied for variety of design purposes. (d) Characteristic focusing feature of utilized scattering regime is not affected by resonant absorption process, providing a high compatibility and integrated trapping mechanism.

absorption further but this additional increment is still considerably lower than our superenhancement (i.e. at least one order of magnitude). We utilize superenhancer nanowire with radius of $1200 \mathrm{~nm}$ in these calculations.

On the enhancer side, if we used same thickness and material of thin film instead of superenhancer wire, we would observe typical thin film interference based enhancement (i.e. multiple interference peaks appears on bare absorption profile) which totally contributes only $4 \%$ in comparison to $700 \%$ of our technique (Figure $2 \mathrm{~b}$, Figure $\mathrm{S} 3 \mathrm{~b})$. Sharp modulation peaks observed in the presence case of enhancer wire $(\mathrm{r}=1200 \mathrm{~nm})$ are associated with unique interference behaviour of cylindrical geometry which involves multiple reflections of light from absorber/enhancer and enhancer/air surfaces interacting with each other to create a distinct pattern of enhanced and diminished intensity bands. Even though contribution of interference-induced absorption enhancement observed in our case is higher than their planar counterparts, still it can also be considered as collateral. Present superenhancer technique supplies versatile conveniences and opportunities to nanowire-based photosensor designs.

Fundamental facilities and practical advantages. Non-resonant scattering of light from low-index structures is highly compatible with resonantly absorbing behaviour of underlying nanowire and the amount of absorption enhancement provided by these enhancer structures can be controlled by size-dependent manner (Figure 3a,b). Increasing the enhancer size yield similar enhancement rates both for TE and TM polarizations. Optimal enhancer size calculated to be around $1500 \mathrm{~nm}$ in its radius which bears $A E$ capacity as high as $\sim 8$ fold (Figure 3c). After this point, total enhancement performance of enhancer wire decreases and at very higher sizes (not shown in the plot), transparent microwire could not function as effective enhancer as in the case of smaller sizes, hence amount of absorption become comparable with provided by bare nanowire itself. Absorption enhancement rates change depending on utilized enhancer size but retains a similar wavelength dependence. Therefore different enhancer size may provide various design opportunities. For instance, smaller sizes are useful when large-area nanowire photovoltaics with area saving is aimed to be achieved. Intermediate nanowire sizes can be used for high-power single nanowire-based compact nanoelectronical systems and very higher sizes can be utilized for construction of novel fiber solar cell schemes.

In contrast to resonant enhancement nature which may induce increments as a local and narrow peaks on absorption spectra, nonresonant characteristics observed in our enhancer structures ensures ultra-broadband enhancement facility and can be applicable both for on-resonance and off-resonance points of Mie absorption phenom- 

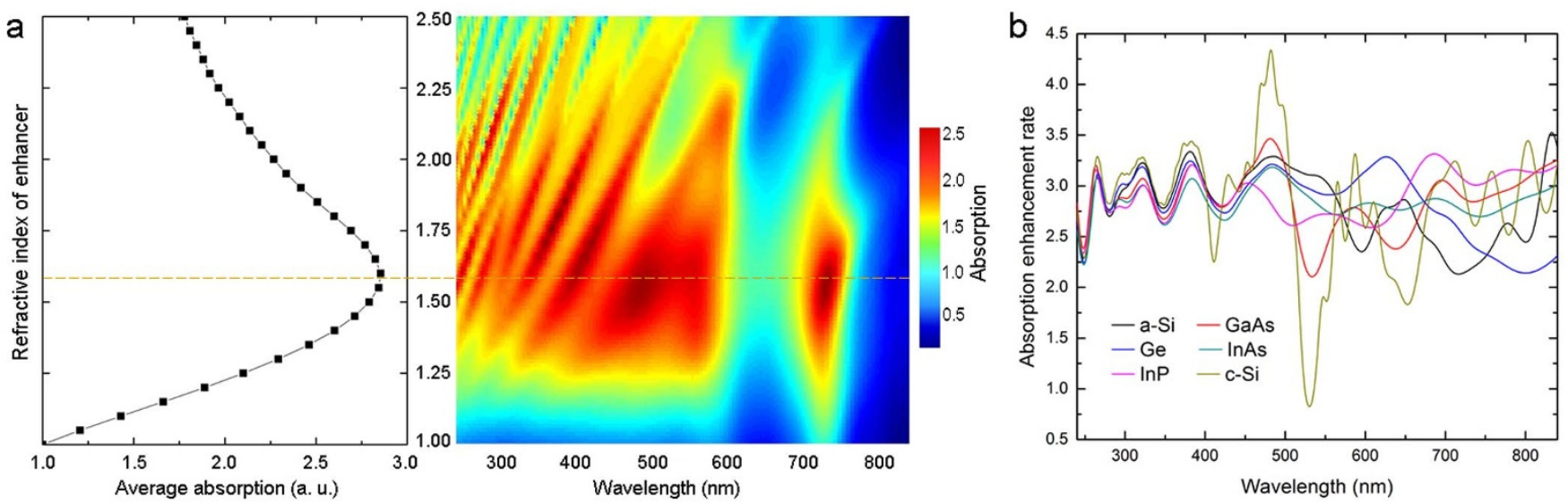

Figure $4 \mid$ Effect of enhancer and absorber material. (a) According to NRM scattering effect, enhancers are required to consist of low refractive index materials. We optimize optical properties of enhancer materials that give maximal absorption efficiency and found that materials with refractive index range of 1.40-1.80 can satisfy this condition. There are broad material options falling within this category including silica glass and polymer materials. (b) As a unique feature of superenhancer concept, it can be applied to any type of absorber material where some of them are expensive as in raw or processed material form but at the same time exhibit more efficient photovoltaics characteristics. Overall $A E$ amount and point-by-point enhancement rates are almost similar for any type of absorber material of fixed size.

enon. However, in order to maximize overall absorption capacity of single nanowire it will be effective to choose nanowire sizes that support efficient leaky-mode resonances simultaneously both for $\mathrm{TE}$ and TM polarizations. Interaction of light with these enhancers can induce spectrally broad (especially for the first scattering order) scattering peaks. This corresponds to very low quality factors $(\mathrm{Q}<1)$ which indicates presence of significantly weak resonant behaviour (Figure S2). Although scattering process is more efficient at the spectral ranges encompassing these broad peaks, the contrast is weak and the overall scattering efficiency is low in comparison to the resonant Mie scattering regime. However, since scattering peak(s) is (are) able to cover visible spectrum as well as edge wavelengths of NIR and UV (even for smaller sizes), then enhancement process can also be effective through major portion of the solar radiation spectra provided that the material attenuation coefficient $(k)$ is also non-zero.

Scattering regimes (e.g. resonant Mie scattering) that suffer from coupling issues alter absorption profile. We observe that even though overall absorption profile remain unaltered during coupling process, field profile of these resonant absorption peaks exhibit an unusual behaviour. For instance, $T M$ polarization modes $\left(\mathrm{TM}_{21}\right.$ and $\left.\mathrm{TM}_{22}\right)$ given in the absorption map possess dissimilar intensity profile than bare nanowire resonances of same size (same holds for TE polarization), which can also be observed from their corresponding field profiles, taking place in $550 \mathrm{~nm}$ and $750 \mathrm{~nm}$ wavelength points respectively (Figure $3 \mathrm{~d}$ ). This may be attributed to incident light profile that is distinct from plane waves utilized in bare nanowire case.

Next critical point is related to polarization-independent manner observed in absorption enhancement. Even though bare nanowires, especially at smaller sizes, cannot efficiently absorb light in TE polarization case, our technique provide a polarization-independent enhancement process, thanks to characteristic feature of described scattering regime. It is worthy to note that, here polarization-independency corresponds to behaviour (i.e. unaltered absorption profile and similar amount of enhancement) that our wire enhance absorption but not makes wire to absorb light in similar spectral profile (as absorber nanowire itself exhibits polarization dependency). Therefore using polarization-independent enhancement instead of polarization-independent absorption is required for avoiding possible confusion. Furthermore, strictly forward scattering and focusing features observed in present technique is also unique to utilized scattering domain and could not be provided by the rest of scattering effects. This induces accumulation of light into very small spot sizes in comparison with the diameter of enhancer and is responsible for enormous absorption enhancement process.

Proposed superenhancer effect also provides unmatched conveniences in terms of material choice. Even though utilized scattering regime is associated with low-refractive index and transparent nanostructures by performing set of FDTD simulations and taking a-Si with $\mathrm{r}=100 \mathrm{~nm}$ as a reference absorber nanowire, we find that superenhancers become even more effective when their refractive indices fall within the range of 1.40-1.80. This range encompasses a broad diversity of polymer materials (e.g. PC, PES, PSU, PS and etc.) as well as many low-index glasses or oxide materials (e.g. $\left.\mathrm{SiO}_{2}\right)$, which can be significantly cheap and abundant compared to solar cell materials. For smaller $n$ values (out of specified range) interaction still exhibit features of present scattering regime though it could not provide as efficient enhancement as the determined range. Above $n=2.00$, optical resonances start to appear in the core region which is reflected as a significant enhancement drop in resulting absorption spectrum (Figure 4a). Such higher refractive indices make nanowire to act as light trapping cavity which become more supportive medium of light, therefore replace forwardly focusing feature of scattered light with multi-directional scattering behaviour (where multi-lobe nearfield profiles of supported resonances determine far-field scattering projections). For better demonstration of Figure 4a (leftmost plot), the abscissa is normalized to the case $n=1.0$ (without enhancer) and in order to obtain average absorption, absorption spectrum is integrated over given spectral range (240-840 nm). Present technique is also highly desirable in terms of applicability to any type of absorber material (Figure $4 \mathrm{~b}$ ). Since working principle of light trapping technique simply relies on characteristic focusing of light into touching underlie nanowire solar cell, then enhancement is expected to occur in entire spectral region where extinction (i.e. absorption coefficient, $k$ ) of absorber material is also non-zero. We test our technique for different solar cell materials including c-Si, InP, GaAs and observed that superenhancers provide material-free enhancement rates, such that overall absorption enhancements are found to be around 3 -fold, independent from absorber material. This provides novel opportunities especially for nanowire solar cells rely on optically and electronically more efficient but less abundant or expensive solar cell materials. Here enhancer and absorber radii are fixed to $100 \mathrm{~nm}$ and $300 \mathrm{~nm}$ respectively.

Additionally, superenhancer concept is effective for different sizes of absorber nanowire. Resonant Mie absorption effect takes place in broad size range of nanowire solar cell which utilize low order(s) of 
leaky modes resonances appearing in smaller sizes where for larger nanowire dimensions number of these supported modes increase. Calculated absorption maps for TE and TM polarizations within 240-840 nm wavelength and $0-150 \mathrm{~nm}$ absorber radii range show contribution of enhancer effect and facilitates comparison between presence (Figure 5a,b) and absence (see Figure S1) cases of enhancer. Enhancers accumulate light into small line spots along longitudinal nanowire axis where absorber is located, and width of this focus is correlated with enhancer size and order of scattering. Therefore in order to obtain maximum performance, absorber size and parameters that affect beam width (e.g. enhancer size) should be selected properly. For fixed enhancer radius of $300 \mathrm{~nm}$, optimum absorber size that is found to give maximum $\mathrm{AE}$ is around $\mathrm{r}=20 \mathrm{~nm}$ for both type of the polarizations (Figure $5 c$ ). Especially in very small sizes of nanowires (i.e. radius of $15-20 \mathrm{~nm}$ ) it is possible to observe very high absorption efficiency for TM polarization, particularly for $\mathrm{TM}_{01}$ mode which utilizes core-concentrated resonant field feature. Together with superenhancer access it is possible to observe $A E$ of 7-fold and we found that this amount corresponds to absorption of bare nanowire with radius over $200 \mathrm{~nm}$, which means 400-fold material conservation in compare to thin film solar cell (if we consider additional 2 -fold enhancement factor of $400 \mathrm{~nm}$ nanowire itself relative to thin film case) (Figure $5 \mathrm{~d}$ ). Since solar radiation is unpolarized (i.e. includes both TE and TM polarization), it is also required to design nanowire absorber for its best absorption performance particularly for photovoltaics applications by choosing proper interplay between size and polarization contributions. For example, we found that it is still possible to save 150 -fold material for unpolarized light case in $r=40 \mathrm{~nm}$ and enhancer size of $r=600 \mathrm{~nm}$, which is open for further optimization. During these calculations we observe that maximal absorption occurs when the absorber to enhancer size ratio is around $1 / 15$.

Proposed superenhancer effect has many practical advantages. For instance, our technique is applicable to any shape of nanowire solar cell and can utilize enhancer with diverse geometries; hence overall it is largely morphology-independent (Figure 6a). Present nanofabrication and nanocharacterization techniques enable the fabrication of a tremendous set of nanowire architectures, which in its low-index or absorbing cases can be used respectively as an enhancer or absorber component of nanowire-based photovoltaics. There are a number of studies that take the structure effects into consideration and investigate their electronic and optical performances for efficient photosensor designs. We take several representative solar cell geometries including cylindrical (characterized so far), hexagonal and square
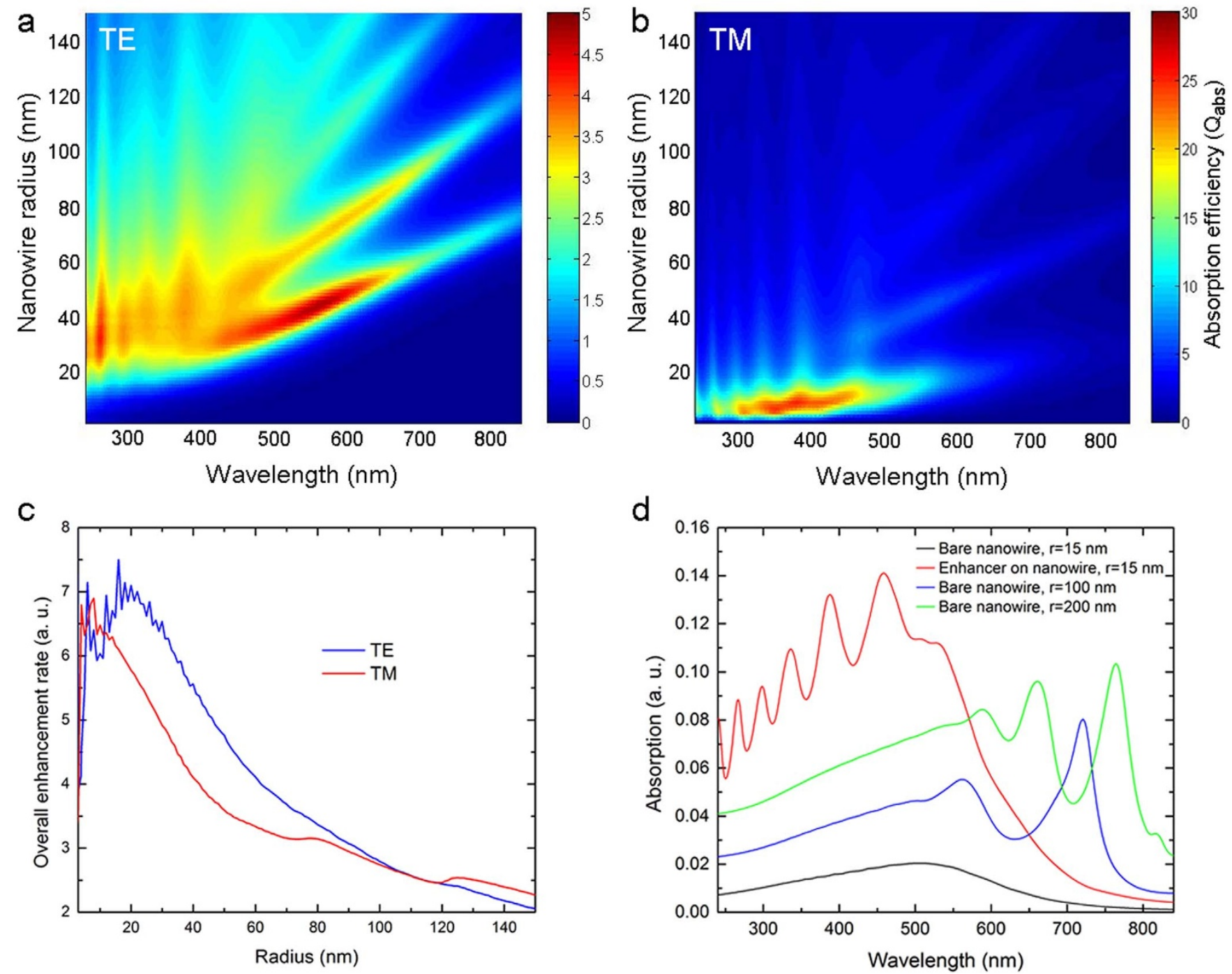

Figure $5 \mid$ Effect of absorber size. Superenhancer technique can be easily implemented to any absorber size as resonant Mie absorption phenomenon is effective in broad nanosize region. (a,b) Map of absorption for TE and TM polarizations facilitates comparison between with and without enhancer cases (see Figure S1). For fixed enhancer size optimal absorber radius is found to be around $20 \mathrm{~nm}$ which gives absorption enhancement capacity as high as 7 -fold. In all these calculations size of enhancer wire fixed to $r=300 \mathrm{~nm}$. 

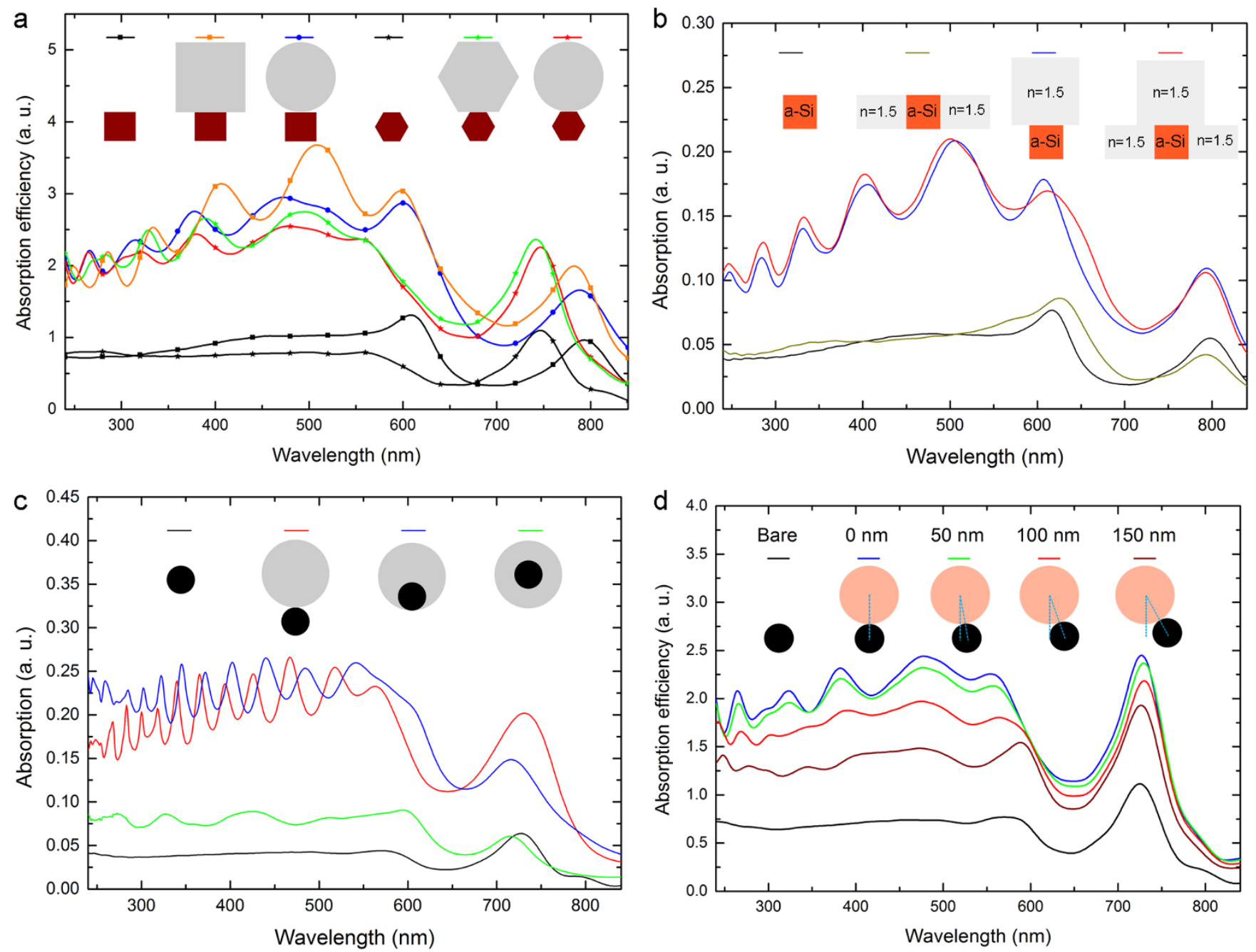

Figure 6 Effect of morphology and fabrication issues. Present fabrication techniques are able to produce a set of distinct regular morphologies some of which are already utilized in the design of nanowire solar cells with their unique absorption performance. Besides cylindrical wire, many further architectures including rectangular and hexagonal absorber and enhancer geometries are also suitable options in 1D nanodevice design and can exhibit resonant Mie absorption and NRM scattering effects as well. Therefore, distinct type of enhancer shapes can be utilized together with various absorber geometries for light trapping purpose which observed to result with similar absorption enhancement amount $\left(A E_{\text {wire_on_sq }}=2.60\right.$, $A E_{\text {sq_on_sq }}=2.80$, $A E_{\text {wire_on_hex }}=2.80, A E_{\text {hex_on_hex }}=2.90$ ). Calculations are performed for TE polarization, but very similar results can be obtained in TM polarization case. (b) Embedding absorber nanowire into low-index medium effectuates similar trapping efficiency as if it would be in air. This facilitates development of superenhancer effect by using lithographic tools. Observed result are same for both cases (i.e. embedded absorber and absorber with air spacing) with values of $A E=2.74$ and $A E=2.65$ respectively. (c) Coating nanowire absorber with conformably manner also yield comparable enhancements results of $A E=4.85$ and $A E=5.15$ for absorber inside and outside cases respectively. On the other hand, well-known core-shell scheme induces only $A E=1.80$. (d) In order to demonstrate practical applicability of our technique, misalignment cases between absorber and enhancer materials are also investigated. Schemes described in the plot (inset) represent these type of (possible) experimental errors where light is incident normally from top. Given values correspond to shifted distance between centers of absorber and enhancer material. Even though AE values reduce when mismatching increases, in general our superenhancer concept is sufficiently tolerable to such experimental errors.

nanowires for observing their absorption performance. In each case, $1 \mathrm{D}$ solar cell geometry exhibits unique resonance behavior within the nanowire absorber where broad resonant absorption peaks shift depending on these morphologies. Placing enhancer wire of similar (i.e. square and hexagonal) or cylindrical shapes on top of these designs yield similar enhancement capacity with all-cylindrical absorber-enhancer case. This feature provide us simplicity during choice of architectural designs for daily life applications. During calculations of absorption efficiency for different geometries, we take radial sizes fix as a $200 \mathrm{~nm}$ (diameter in wire case and side values in hexagonal and square cases). In order to obtain absorption efficiency for non-circular wires, we divide absorption cross-section directly with these side values. Therefore obtained efficiency values for these non-circular cases are arbitrary (but very close to their real efficiency values) and mainly emphasize relative absorption capacity for the presence and absence cases of our enhancer apparatus.

Regarding to applicability of superenhancer concept, there are many proper fabrication options with their distinct set of useful features. One potential way to this end is utilizing lithographic tools. It is possible to define proper material pattern on substrate (enhancer on absorber). Since any of the 1D shape is applicable as an absorber or enhancer, then we can define rectangular $1 \mathrm{D}$ array with rectangular enhancer wire on it. We found that embedding of absorber in low-index medium $(n=1.5)$ and air (Figure $6 \mathrm{~b}$ ) yield similar enhancement results, hence any of these schemes can be used for the observation of the described effect (i.e. respectively $A E=2.74$ and $A E=2.65$ are observed for enhancer square with side size of $600 \mathrm{~nm}$ and absorber square with side size of $200 \mathrm{~nm}$ ). This fabrication 

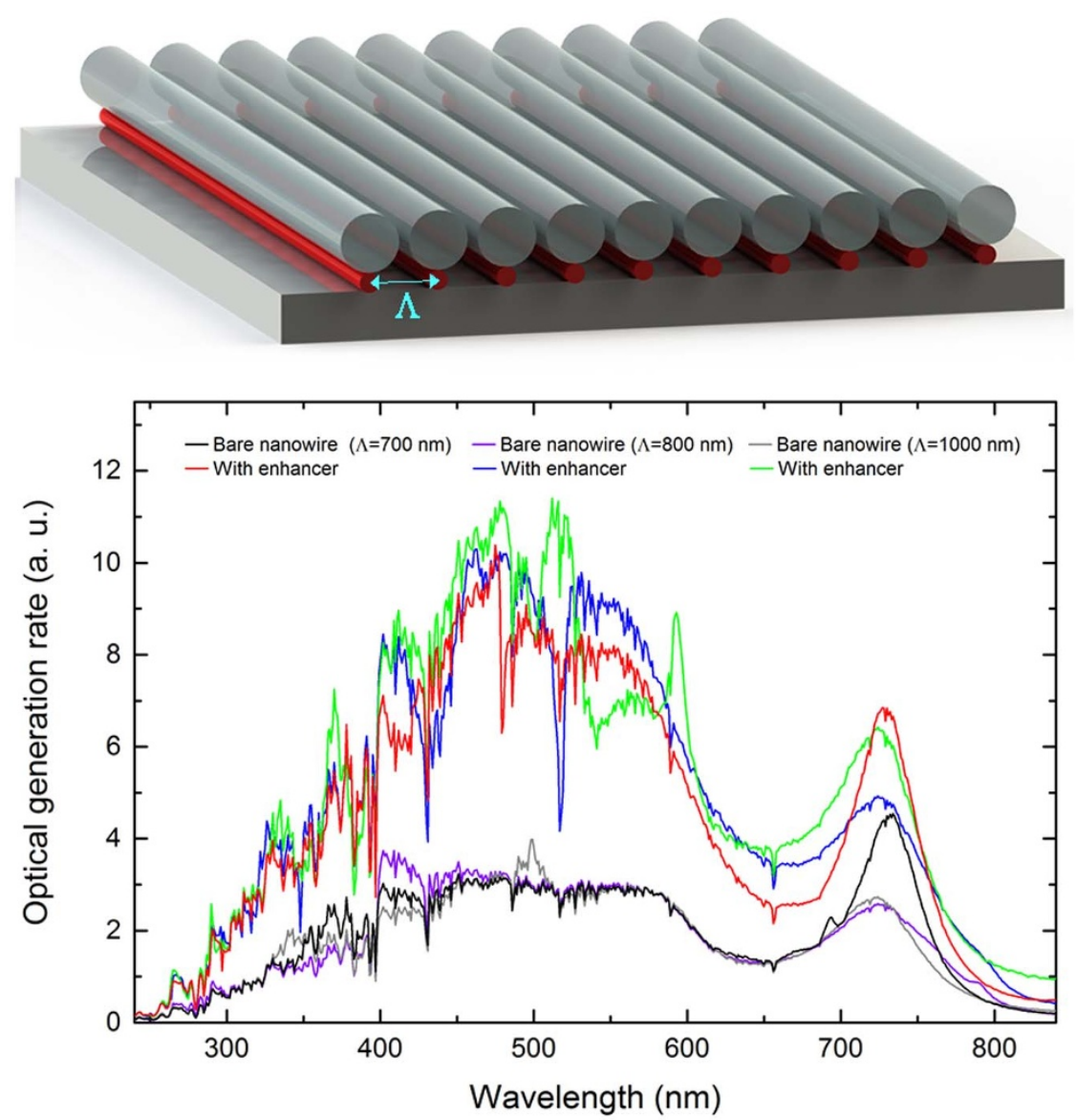

Figure $7 \mid$ Large-area scaling. For large-area applications nanowire solar cells are required to be designed as efficient arrays. Bare nanowire arrays display maximum absorption performance for pitch sizes of $600 \mathrm{~nm}-700 \mathrm{~nm}$ (Figure S4). Adding enhancer layer on this array result with similar absorption enhancement features as it would be a single enhancer-absorber dual. By using optimal design parameters, it is possible to decrease required installation area $>1.5$-fold. Calculated AM1.5 integrated optical generation rates are 2.33 for $\Lambda=700,2.61$ for $\Lambda=800$, and 2.87 for $\Lambda=1000 \mathrm{~nm}$. TE polarization is used for demonstration. Similar results can be observed for TM polarization case.

method can be further optimized to be more efficient and simple in its design (such as utilizing soft molds for transferring absorber on enhancer scheme to its inverse required form).

The second useful method is associated with the implementation of deposition techniques (e.g. CVD). We observe that, after obtaining absorber nanowires by using any of bottom-up or top-down fabrication approaches, it is possible to coat absorber nanowire surface conformably with any low-index enhancer up to nonconcentric configuration (absorber-embedded enhancer) similar as in Figure $6 \mathrm{c}$ (inset) is obtained. Such off-centered coating observed to yield even better $A E$ result (i.e. $A E_{\text {out }}=4.85<A E_{\text {in }}=5.15$ ) than the absorber in the outer edge case (for both cases size of enhancer nanowire taken as $\mathrm{r}=600 \mathrm{~nm}$ and absorber nanowire as $\mathrm{r}=100 \mathrm{~nm}$ ) are utilized. Especially utilizing suspended nanowire scheme for coating process will definitely facilitate observation of the described effect $^{36,37}$. Here, it is also useful to compare absorption enhancement performance of our design with frequently utilized core-shell scheme. Our case can be considered as core-shifted version of core-shell scheme where either "core in the outer edge" or "core at the inner edge" schemes supply at the very least 5-fold more effective $A E$ than "core in the center" (core-shell) scheme (Figure 6c).

Regarding to large-area facility (as well as many other unique features) it will be effective to utilize new member of top-down approach, called iterative size reduction technique (ISR) ${ }^{38}$. This method provides macroscale control where final nanostructure configuration can be determined at larger sizes. Silica/polymer on absorber scheme can be designed starting from $\mathrm{cm}$-scale sizes and as a result of two step fabrication process nanoscale counterpart of described scheme can be achieved. This method is also convenient for separate fabrication of large-area monolayer nanowire absorbers and enhancers which later can be matched for its maximal performance provided by easy-handling feature of the method. Especially in latter case, we calculated that the present trapping design is also tolerable to absorber to enhancer mismatching in a reasonable extent. We perform mismatching simulations for cylindrical cases; however, results are clearly applicable to any of the present $1 \mathrm{D}$ shape. In small shift cases absorption enhancement observed to remain significantly unaltered and even in larger dislocations we can get significant absorption enhancement (Figure 6d). Hereby, many present nanofabrication methods with their suitable features will facilitate the widespread implementation of our proposed technique.

So far by taking advantages of our approach we concentrate on the design of efficient single nanowire solar cells for the operation of nanoelectronics or self-powered nanosystems. Next important characteristic of the described technique is its applicability to large areas (Figure 7). Here we found that, it is possible to obtain highly efficient large-area photovoltaics systems by using properly engineered enhancer sizes. For instance, at the expense of larger installation area, but few order of less material amount, it is possible to construct highpower photovoltaics systems. However when area preservation is the main concern, design parameters (e.g. enhancer diameter, pitch size) required to be changed. According to theoretical calculations, nanowire array exhibit its best absorption performance when it has pitch sizes of 600-700 nm (Figure S4). This is due to overlapping of these 
spacing values with one or more of Mie resonances present within the absorber nanowire. By adding proper size $(\mathrm{r}=300 \mathrm{~nm})$ of superenhancers on absorber nanowire array and utilizing optimal pitch sizes, we observe 2.33-fold increment in absorption amount. By taking advantage of proper arraying that yields higher absorption in comparison to nanowire alone $(>1.1$ fold) and further $>2.1$-fold enhancement of nanowire itself to thin-film scheme and dividing these products by 3.5 (thickness of thin film $700 \mathrm{~nm}$ (pitch size) is divided by nanowire width $200 \mathrm{~nm}$ within the same pitch), we can obtain $>1.5$ fold area preservation compared to thin film solar cells. Taking the different effects into account and further optimizations will definitely promote these findings even up to higher values.

\section{Discussion: Versatile opportunities}

We provide unique light accumulation nanotool, called "superenhancers", for designing efficient nanowire-based photosensor devices. This technique utilizes exclusive features of NRM scattering effect including broadband enhancement, large-area accumulation of light along nanowires, unique field focusing, polarization independency, forward scattering and distinctive coupling behaviour which are recognized as ideal light trapping characteristics. We demonstrate that our technique is able to provide crucial material conservation exceeding two orders of magnitude $\left(>10^{2}\right)$, giant power amplification $(A E \%=1500 \%)$ and is useful for large-area applications with critical area saving. Unlike resonant trapping systems, where enhancement may depend on intrinsic (material-dependent) or extrinsic (size-dependent) features of nanowire, in this technique NRM scattering provides an enhancement profile largely independent from the type of material and nanowire size. Furthermore, superenhancers that are optimally required to be low-refractive index materials including many polymer materials and some well-known glasses or oxides (e.g. silica) are cheap and abundant materials and also reached maturity in their nanoscale production. Therefore, we believe our superenhancer concept will present new opportunities for nanowire-based photovoltaics designs especially which utilize more efficient absorber materials but are less abundant or expensive.

Superenhancer effect can also take place in variety of device designs and potential to provide versatile opportunities in different circumstances. For instance, larger sizes of enhancer are exclusively useful and promising for highly-efficient fiber solar cell design ${ }^{39}$. Therefore, by utilizing absorber nanowire at the inner edge of enhancer (see Figure 6c) with proper sizes it is possible to fabricate textilecompatible high-power source for fabric-integrated photovoltaics. Furthermore, it is open to question whether it is possible to choose material to serve as both enhancer and fabric. Nanowire solar cells are also expected to be an integral part of self-powered nanosystems ${ }^{40,41}$ which are promising as nanosensors for monitoring health or serving as versatile nanoprocessors. Correct interplay between superenhancer size and its efficiency can also be utilized in the design of highly compact power source that could be integrated with nanoelectronics. Moreover, superenhancer concept is highly compatible with flexible solar cells and applicable for on-chip solar cell designs as well.

Superenhancer concept is not restricted to solar cell devices alone but can be used in development of many optoelectronic devices including phototransistors, photodetectors and etc. Especially harnessing our technique in IR region can provide powerful solutions for low light intensity problem of IR photodetectors. We believe there is a great deal of work on this area and even better absorber performance can be obtained using different designs, such as utilizing solar superabsorbers $^{42}$ together with our method potential to fully satisfy nanowire solar cells in terms of absorption capacity.

\section{Methods}

FDTD Simulations. We utilize simulation tool of finite difference time domain (FDTD) method (commercialized by Lumerical Solutions Inc.) which relies on numerical solutions of differential Maxwell equations and take almost all realistic possibilities into consideration without obligation of assumptions or approximations (even though they are also possible), hence providing a very powerful and commonly accepted technique for scientific purposes. We investigate absorption and scattering characteristics of nanowire geometries in $2 \mathrm{D}$ simulation domains considering their very high aspect ratios. Total field scattered field (TFSF) source is utilized for effective absorption and scattering analysis by its characteristics feature of separating fields into interacting and non-interacting field domains. Particularly in performing of large-area simulations we utilize plane-wave source. In both type of source we set incident source as broadband in the $240-840 \mathrm{~nm}$ wavelength range. In order to collect scattering light or detect absorbed light within the absorber nanowire region we utilize rectangular analysis loop consist of frequency-domain power monitors and place them outside and inside region of TFSF source respectively. In order to obtain mode profiles we utilize frequency-domain profile monitors. Boundary conditions are set as symmetric and asymmetric in cylindrical symmetric cases, periodic boundary conditions for large-area calculations and perfectly matched layer (PML) boundary conditions in all remaining cases. Refractive indices of enhancer material set to 1.50 as a reference in most of simulations (except Figure 4a). Absorber material set as a-Si. According to these references absorption coefficient is non-zero up to wavelength of $840 \mathrm{~nm}$. During simulations of other absorber materials (Figure 4b), we utilize material database provided by Lumerical software. In all simulations we perform material fit appropriately and ignore underlying substrate which have insignificant effect for presence and absence cases of our enhancer wire.

1. Kempa, T. J. et al. Semiconductor nanowires: a platform for exploring limits and concepts for nano-enabled solar cells. Energy Environ. Sci. 6, 719-733 (2013).

2. Garnett, E. C., Brongersma, M. L., Cui, Y. \& McGehee, M. D. Nanowire solar cells Ann. Rev. Mater. Res. 41, 269-295 (2011).

3. Tang, J., Huo, Z., Brittman, S., Gao, H. \& Yang, P. Solution-processed core-shell nanowires for efficient photovoltaic cells. Nat. Nanotechnol. 6, 568-572 (2011).

4. Wallentin, J. et al. InP nanowire array solar cells achieving $13.8 \%$ efficiency by exceeding the ray optics limit. Science 339, 1057-1060 (2013).

5. Krogstrup, P. et al. Single-nanowire solar cells beyond the Shockley-Queisser limit. Nature Photon. 7, 306-310 (2013).

6. LaPierre, R. R. et al. III-V nanowire photovoltaics: Review of design for high efficiency. Phys. Status Solidi RRL 7, 815-830 (2013).

7. Shah, A., Torres, P., Tscharner, R., Wyrsch, N. \& Keppner, H. Photovoltaic technology: the case for thin-film solar cells. Science 285, 692-698 (1999).

8. Aberle, A. G. Thin-film solar cells. Thin Solid Films 517, 4706-4710 (2009).

9. Tian, B., Kempa, T. J. \& Lieber, C. M. Single nanowire photovoltaics. Chem. Soc. Rev. 38, 16-24 (2009).

10. Cho, H. D. et al. Photovoltaic device on a single $\mathrm{ZnO}$ nanowire $\mathrm{p}-\mathrm{n}$ homojunction. Nanotechnology 23, 115401 (2012).

11. Kind, H. et al. Nanowire ultraviolet photodetectors and optical switches. $A d v$. Mater. 14, 158-160. (2002).

12. Fan, Z. et al. Large-scale, heterogeneous integration of nanowire arrays for image sensor circuitry. PNAS 105, 11066-11070 (2008).

13. Wong, W. S. et al. Hybrid Si nanowire/amorphous silicon FETs for large-area image sensor arrays. Nano Lett. 11, 2214-2218 (2011).

14. Ul Hasan, K. et al. Single nanowire-based UV photodetectors for fast switching. Nanoscale Res. Lett. 6, 1-6 (2011)

15. Wang, H. High gain single GaAs nanowire photodetector. Appl. Phys. Lett. 103, 093101 (2013).

16. Chen, G. et al. Single-Crystalline p-Type Zn3As2 Nanowires for field-effect transistors and visible-light photodetectors on rigid and flexible substrates. $A d v$. Funct. Mater. 23, 2681-2690 (2013)

17. Brongersma, M. L., Cui, Y. \& Fan, S. Light management for photovoltaics using high-index nanostructures. Nat. Mater. 13, 451-460 (2014).

18. Kim, S. K. et al. Design of nanowire optical cavities as efficient photon absorbers. ACS Nano 8, 3707-3714 (2014).

19. Cao, L. et al. Engineering light absorption in semiconductor nanowire devices. Nat. Mater. 8, 643-647 (2009).

20. Kim, S. K. et al. Tuning light absorption in core/shell silicon nanowire photovoltaic devices through morphological design. Nano Lett. 12, 4971-4976 (2012).

21. Liu, W. F., Oh, J. I. \& Shen, W. Z. Light absorption mechanism in single c-Si (core)/a-Si (shell) coaxial nanowires. Nanotechnology 22, 125705 (2011).

22. Xie, W. Q., Liu, W. F., Oh, J. I. \& Shen, W. Z. Optical absorption in c-Si/a-Si: H core/shell nanowire arrays for photovoltaic applications. Appl. Phys. Lett. 99, 033107 (2011).

23. Kallel, H., Chehaidar, A., Arbouet, A. \& Paillard, V. Enhanced absorption of solar light in Ge/Si core-sheath nanowires compared to Si/Ge core-sheath and SilxGex nanowires: A theoretical study. J. Appl. Phys. 114, 224312 (2013).

24. Liu, W. F., Oh, J. I. \& Shen, W. Z. Light trapping in single coaxial nanowires for photovoltaic applications. Electron Device Lett. IEEE 32, 45-47 (2011).

25. Liu, W. Tunable light absorption of Si nanowires by coating non-absorbing dielectric shells for photovoltaic applications. Optoelectron. Adv. Mater. 5, 916-919 (2011)

26. Hua, X., Zeng, Y. \& Shen, W. Z. Semiconductor/dielectric half-coaxial nanowire arrays for large-area nanostructured photovoltaics. J. Appl. Phys. 115, 124309 (2014). 
27. Colombo, C., Krogstrup, P., Nygård, J., Brongersma, M. L. \& i Morral, A. F. Engineering light absorption in single-nanowire solar cells with metal nanoparticles. New J. Phys. 13, 123026 (2011)

28. Khudiyev, T., Huseyinoglu, E. \& Bayindir, M. Non-resonant Mie scattering: Emergent optical properties of core-shell polymer nanowires. Sci. Rep. 4, 4607; DOI:10.1038/srep04607 (2014).

29. Van de Hulst, H. C. Light scattering by small particles. (Courier Dover Publications, 2012).

30. Papavassiliou, G. C. Optical properties of small inorganic and organic metal particles. Prog. Solid State Chem. 12, 185-271 (1979).

31. Fan, X., Zheng, W. \& Singh, D. J. Light scattering and surface plasmons on small spherical particles. Light Sci. Appl. 3, e179 (2014).

32. Khudiyev, T., Ozgur, E., Yaman, M. \& Bayindir, M. Structural coloring in large scale core-shell nanowires. Nano Lett. 11, 4661-4665 (2011).

33. Bohren, C. F. \& Huffman, D. R. Absorption and scattering of light by small particles (John Wiley \& Sons Inc.: New York, 1998).

34. Schuller, J. A. et al. Plasmonics for extreme light concentration and manipulation. Nat. Mater. 9, 193-204 (2010).

35. Shen, S. C. \& Huang, J. C. Rapid fabrication of a micro-ball lens array by extrusion for optical fiber applications. Opt. Express 17, 13122-13127 (2009).

36. Lee, K. N. et al. Fabrication of suspended silicon nanowire arrays. Small 4, 642-648 (2008).

37. San Paulo, Á. et al. Suspended mechanical structures based on elastic silicon nanowire arrays. Nano Lett. 7, 1100-1104 (2007).

38. Yaman, M. et al. Arrays of indefinitely long uniform nanowires and nanotubes. Nat. Mater. 10, 494-501 (2011).

39. He, R. et al. Silicon p-i-n Junction Fibers. Adv. Mater. 25, 1461-1467 (2013).

40. Xu, S., Qin, Y., Xu, C., Wei, Y., Yang, R., \& Wang, Z. L. Self-powered nanowire devices. Nat. Nanotechnol. 5, 366-373 (2010).

41. Tian, B. et al. Coaxial silicon nanowires as solar cells and nanoelectronic power sources. Nature 449, 885-889 (2007).
42. Yiling, Y., Lujun, H. \& Linyou, C. Semiconductor solar superabsorbers. Sci. Rep. 4, 4107; DOI:10.1038/srep04107 (2014).

\section{Acknowledgments}

We would like to thank Erol Ozgur and Tamer Dogan for critical reading of the manuscript and fruitful discussions. The research leading to these results has received funding from the European Research Council under the European Union's Seventh Framework Programme (FP/2007-2013)/ERC Grant Agreement n. 307357. M.B. acknowledges partial support from the Turkish Academy of Sciences (TUBA).

\section{Author contributions}

T. K. and M. B. designed and carried out research, analyzed data and wrote the paper.

\section{Additional information}

Supplementary information accompanies this paper at http://www.nature.com/ scientificreports

Competing financial interests: The authors declare no competing financial interests.

How to cite this article: Khudiyev, T. \& Bayindir, M. Superenhancers: Novel opportunities for nanowire optoelectronics. Sci. Rep. 4, 7505; DOI:10.1038/srep07505 (2014).

This work is licensed under a Creative Commons Attribution-NonCommercialShareAlike 4.0 International License. The images or other third party material in this article are included in the article's Creative Commons license, unless indicated otherwise in the credit line; if the material is not included under the Creative Commons license, users will need to obtain permission from the license holder in order to reproduce the material. To view a copy of this license, visit http:// creativecommons.org/licenses/by-nc-sa/4.0/ 